Portland State University

PDXScholar

Electrical and Computer Engineering Faculty

Publications and Presentations

2-1-1979

\title{
Scattering of focused beams by tenuous particles
}

S. Colak

Cavour W. Yeh

Lee W. Casperson

Portland State University

Follow this and additional works at: https://pdxscholar.library.pdx.edu/ece_fac

Part of the Electrical and Computer Engineering Commons

Let us know how access to this document benefits you.

\section{Citation Details}

S. Colak, C. Yeh, L. W. Casperson . Scattering of focused beams by tenuous particles. Applied Optics, Volume 18, Number 3 (February 1979), pp. 294-302.

This Article is brought to you for free and open access. It has been accepted for inclusion in Electrical and Computer Engineering Faculty Publications and Presentations by an authorized administrator of PDXScholar. Please contact us if we can make this document more accessible: pdxscholar@pdx.edu. 


\title{
Scattering of focused beams by tenuous particles
}

\author{
S. Colak, C. Yeh, and L. W. Casperson
}

\begin{abstract}
This paper deals with the problem of the scattering of focused laser beams by tenuous particles using an iterative technique. The results are shown to be accurate provided that (a) the polarizability of the particle medium is small and (b) the phase shift of the central ray is less than 2. It was found that when the size of the incident beam waist is close to that of the scatterer, the scattered field deviates significantly from that for the incident plane wave case. Specific examples are given.
\end{abstract}

\section{Introduction}

By strongly focusing a laser beam in a polydisperse ensemble medium, we have shown that ${ }^{1}$ it is possible to obtain the scattering characteristics of individual particles in such a medium. In order to make use of this focused beam technique to deduce the physical properties of individual particles within an ensemble, the relationships between the scattered fields and the particle properties must be known in advance. In a previous paper, ${ }^{2}$ we have derived an explicit analytical formula for the scattered field of a particle in a focused beam under the Rayleigh-Gans approximation. This approximation requires that the following two criteria be satisfied: (1) the relative refractive index $n$ for the scatterer with respect to its surrounding medium is close to 1 , that is, $|n-1| \ll 1$, and (2) the phase shift $\delta$ of the central ray through the particle is small, $\delta=2 k a|n-1|$ $\ll 1$, where $k$ is the propagation constant, and $a$ is a typical particle dimension. It should be recognized that these criteria are very restrictive. The purpose of this paper is to extend the iterative technique developed by Shifrin $^{3}$ and later applied by Acquista ${ }^{4}$ for the treatment of the plane wave scattering problem to the focused beam scattering problem. This iterative technique will yield results which will be valid under two broader criteria: (1) the polarizability $\alpha$ is small, $\alpha=$ $\left[3\left(n^{2}-1\right)\right] /\left[4 \pi\left(n^{2}+2\right)\right] \ll 1$, and (2) the phase shift $\delta$ of the central ray is less than 2 . Several numerical examples will be given.

The authors are with University of California, Los Angeles, Electrical Sciences \& Engineering Department, Los Angeles, California 90024.

Received 14 July 1978.

0003-6935/79/03294-09\$00.50/0.

(C) 1979 Optical Society of America.

\section{Formulation of the Problem}

The geometry of the problem is shown in Fig. 1. A tenuous particle whose physical parameters satisfy the necessary broader criteria given in Sec. I is situated in an incident focused Gaussian beam. We are interested in the scattered field.

The transverse electric field of an incident Gaussian beam takes the form ${ }^{5}$

$$
\mathbf{E}_{b}(x, y, z, t)=\left(A_{x} \hat{x}_{x}+A_{y} \hat{y}_{y}\right) f(x, y, z) \exp (i \omega t),
$$

where

$$
\begin{aligned}
f(x, y, z)= & \frac{W_{0}}{W(z)} \exp (-i k z) \exp \left[-i k\left(x^{2}+y^{2}\right) / 2 R(z)\right] \\
& \cdot \exp \left[-\left(x^{2}+y^{2}\right) / W^{2}(z)\right] \\
& \cdot \exp \left[-i \arctan \left(z / z_{0}\right)\right], \\
W(z)= & W_{0}\left[1+\left(z^{2} / z_{0}^{2}\right)\right]^{1 / 2}, \\
R(z)= & z\left[1+\left(z_{0}^{2} / z^{2}\right)\right], \\
z_{0}= & \pi W_{0}^{2} / \lambda .
\end{aligned}
$$

The quantities $W(z)$ and $R(z)$ are, respectively, the spot size and the radius of curvature of the phase front of the beam at $z . \quad A_{x}$ or $A_{y}$ are, respectively, the complex amplitude constant of the electric field vectors polarized in the $x$ or $y$ direction. $W_{0}$ is the beam waist spot size, and $z_{0}$ is the Rayleigh length of the beam. $\omega$ is the frequency of the wave, and $k=\omega\left(\epsilon_{0} \mu_{0}\right)^{1 / 2}$ is the wavenumber. The relations given above assume that the longitudinal $z$ component of the beam field is much smaller than the transverse components. This assumption does not cause any error greater than $2.5 \times$ $10^{-3}$ in the transverse components given by Eq. (1) even in the most sharply focused beam cases. ${ }^{2}$

Instead of solving the problem for the complicated beam expression of Eq. (1), this work will involve a more precise and practical method, which makes use of the plane wave spectrum (PWS) of the incident beam. The PWS of the beam is obtained as follows: the beam expression given by Eq. (1) can be Fourier transformed 


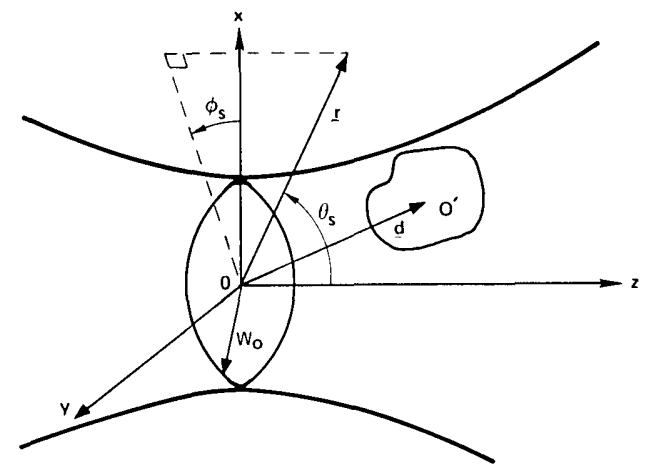

Fig. 1. Geometry of the scattering problem. $W_{0}$ is the spot size of the beam which is centered on the $O$ system. The beam propagates along the $+z$ direction. The scatterer is centered on the $O^{\prime}$ system, which is displaced from the $O$ system by $\mathbf{d}=\left(d_{x}, d_{y}, d_{z}\right)$, and it may also be rotated with respect to the $O$ system. $\theta_{s}$ and $\phi_{s}$ define the scattering angles.

(spatially) in the $z=0$ plane to yield the spectrum functions of the beam field components, which are

$$
\begin{aligned}
S_{x}(p, q) & =\frac{A_{x}}{\lambda^{2}} \iint_{-\infty}^{+\infty} f(x, y, 0) \exp [i k(p x+q y)] d x d y \\
& =A_{x}\left[\left(b^{2}\right) / \pi\right] \exp \left[-b^{2}\left(p^{2}+q^{2}\right)\right], \\
S_{y}(p, q) & =\frac{A_{y}}{\lambda^{2}} \iint_{-\infty}^{+\infty} f(x, y, 0) \exp [-i k(p x+q y)] d x d y \\
& =A_{y}\left[\left(b^{2}\right) / \pi\right] \exp \left[-b^{2}\left(p^{2}+q^{2}\right)\right],
\end{aligned}
$$

where

$$
b=k W_{0} / 2 \text {. }
$$

The inverse transform gives the $x$ and $y$ polarized incident beam electric fields at the $z=0$ plane:

$E_{b x}(x, y, 0)=A_{x} f(x, y, 0)=\iint_{-\infty}^{+\infty} S_{x}(p, q) \exp [i k(p x+q y)] d p d q$,

$E_{b y}(x, y, 0)=A_{y} f(x, y, 0)=\iint_{-\infty}^{+\infty} S_{y}(p, q) \exp [i k(p x+q y)] d p d q$,

where the time dependence, $\exp (i \omega t)$, of the fields is omitted. The scalar Helmholtz equation,

$$
\begin{aligned}
\nabla^{2} E_{b j}(x, y, z)+k^{2} E_{b j}(x, y, z) & =0, \\
j & =x, \text { or } y,
\end{aligned}
$$

together with the boundary conditions given by Eq. (3) are used ${ }^{6}$ to find the spectrum of the incident fields everywhere in space. Thus, for a wave propagating in the positive $z$ direction, the following equations for the transverse incident beam components are obtained:

$$
\begin{aligned}
& E_{b x}(x, y, z)=\iint_{-\infty}^{+\infty} S_{x}(p, q) \exp [i k(p x+q y-s z)] d p d q, \\
& E_{b y}(x, y, z)=\iint_{-\infty}^{+\infty} S_{y}(p, q) \exp [i k(p x+q y-s z)] d p d q,
\end{aligned}
$$

where

$$
s=\left(1-p^{2}-q^{2}\right)^{1 / 2} .
$$

The plane wave spectrum of the $z$ component of the incident electric field is found using the divergence condition $\nabla \cdot \mathbf{E}_{b}=0$. Finally, the PWS of the total incident electric field is found by summing all three electric field expressions corresponding to three different polarizations and by using Eq. (2). The result is

$$
\begin{aligned}
\mathbf{E}_{b}(x, y, z,= & \frac{b^{2}}{\pi} \iint_{-\infty}^{+\infty}\left(A_{x} \hat{x}+A_{y} \hat{y}+\frac{p A_{x}+q A_{y}}{s} \hat{z}\right) \\
& \cdot \exp \left[-b^{2}\left(p^{2}+q^{2}\right)\right] \exp [i k(p x+q y-s z)] d p d q,
\end{aligned}
$$

where $s=\left(1-p^{2}-q^{2}\right)^{1 / 2}$, and $b=k W_{0} / 2$. Similar derivations have been carried out by $\mathrm{Clemmow}^{7}$ and Carter. ${ }^{8}$ It is seen that the integrand in Eq. (6) can be viewed as a form of plane wave with amplitudes

$$
\mathbf{A}_{i}(p, q)=\left(A_{x} \hat{x}+A_{y} \hat{y}+\frac{p A_{x}+q A_{y}}{s} \hat{z}\right) \exp \left[-b^{2}\left(p^{2}+q^{2}\right)\right]
$$

and propagation vectors

$$
\mathbf{k}_{i}(p, q)=k(p \hat{x}+q \hat{y}-s \hat{z}) .
$$

Hence, each plane wave component in the integrand can be represented by the general form

$$
\mathbf{E}_{i}(p, q)=\mathbf{A}_{i}(p, q) \exp \left[i \mathbf{k}_{i}(p, q) \cdot \mathbf{r}\right] .
$$

The expression of Eq. (6) is called the PWS (plane wave spectrum) of the incident laser beam. The plane wave components of this spectrum have polarization and propagation vectors as defined by Eqs. (7) and (8), and they can be expressed in a compact form as shown in Eq. (9). If the scattering problem is solved for one of these plane wave components, the total scattering field pattern due to the incident laser beam will be found by summing over a continuous superposition of such solutions. Of course, this procedure is true for linear phenomenon only.

As mentioned earlier, Shifrin ${ }^{3}$ developed an integro-differential equation, which gives the effective field in the presence of a tenuous scatterer. Later, Acquista, ${ }^{4}$ by expanding the effective field and the scattered fields in the power series of the polarizability $\alpha$ and by making use of the Fourier transforms, obtained the scattered field expressions for the first two iterations. They are summarized as follows:

$$
\begin{aligned}
\mathbf{E}_{s}^{(1)}(\mathbf{r})= & \frac{k^{2}}{r} \exp (i k r) \mathbf{E}_{o \perp} u(k \hat{r}-\mathbf{k}), \\
\mathbf{E}_{s}^{(2)}(\mathbf{r})= & \frac{2 k^{2}}{(2 \pi)^{2}} \frac{\exp (i k r)}{r} \int_{V_{m}} \frac{u(\mathbf{m}+k \hat{r}) u(\mathbf{m}+\mathbf{k})}{m^{2}-k^{2}} \\
& \left.\cdot\left[\left(\frac{2}{3} k^{2}+\frac{1}{3} m^{2}\right) \mathbf{E}_{o \perp}-\left(\mathbf{m} \cdot \mathbf{E}_{o}\right) \mathbf{m}_{\perp}\right]\right] d^{3} m, \\
\mathbf{E}_{s}(r)= & \alpha \mathbf{E}_{s}^{(1)}(\mathbf{r})+\alpha^{2} \mathbf{E}_{s}^{(2)}(\mathbf{r}),
\end{aligned}
$$

where $\mathbf{E}_{s}$ is the scattered field, and $\mathbf{E}_{s}^{(1)}$ and $\mathbf{E}_{s}^{(2)}$ are the scattered field in the first and second iterations, respectively. Note that the above equations were derived assuming that the incident wave is a single plane wave with vector amplitude $\mathbf{E}_{o}$ and propagation vector $\mathbf{k}$. The symbols in the above equations are defined as follows: $u(\mathbf{v})$ is the spatial Fourier transform of the 3-D pupil function $u(\mathbf{r})$ of the scatterer, which is given by 


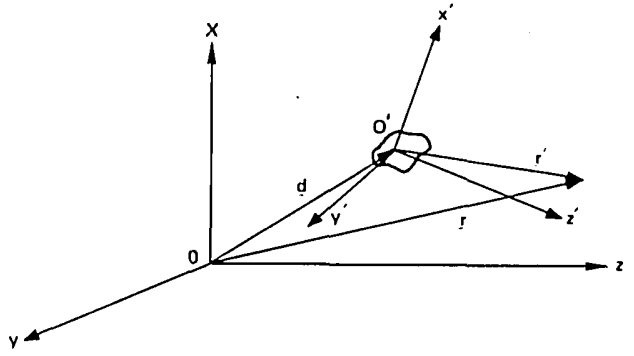

Fig. 2. Placement of the rotated scatterer coordinate system $O^{\prime}$ with respect to the stationary beam system $O . \mathbf{r}$ and $\mathbf{r}^{\prime}$ are arbitrary position vectors in the beam and the scatterer system, respectively. d is the vector defining the displacement of the scatterer from the origin of the beam system.

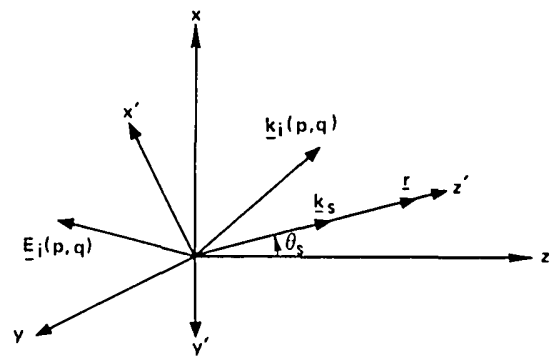

Fig. 3. Diagram for coordinate transformations. $\mathbf{k}_{s}, \mathbf{r}$, and $\hat{z}^{\prime}$ are vectors all directed to the observation point. The incident field propagation vector $\mathbf{k}_{i}(p, q)$ is in the $x^{\prime} z^{\prime}$ plane. The scattering plane is chosen to be the $x z$ plane, and thus the vector $\mathbf{k}_{s}$ (also $\mathbf{r}$ and $\hat{z}^{\prime}$ ) is in this plane, and it makes a scattering angle $\theta_{s}$ with the $z$ axis of the beam system $O$.

$$
u(\mathbf{v})=\int_{V_{s}} \exp (i \mathbf{v} \cdot \mathbf{r}) d^{3} r,
$$

where $V_{s}$ means that the integration is to be carried out over the total scatterer volume; $\mathbf{m}$ and $d^{3} m$ are, respectively, the vectors and the volume elements of the 3-D spatial frequency domain; $V_{m}$ indicates that the integration in this domain is to be carried out over all space; $\mathbf{E}_{o \perp}$ is the incident plane wave vector amplitude component perpendicular to the scattered field propagation vector which is along $\mathbf{r}$ as shown in Fig. 1; the polarizability $\alpha$ of the scatterer material is given by

$$
\alpha=\frac{3}{4 \pi}\left(\frac{n^{2}-1}{n^{2}+2}\right)
$$

where $n$ is the index of refraction of the scatterer.

Acquista's results given by Eq. (9) were obtained by assuming a rotated coordinate system (scatterer system) whose $z^{\prime}$ axis was along the vector $\mathbf{r}$, which defined the scattering direction in the laboratory coordinate system (beam system). He also chooses the $x^{\prime}$ axis of his coordinate system such that the propagation vector of the incident plane wave lies in the $x^{\prime} z^{\prime}$ plane of the scatterer system. The relations between the scatterer and the beam systems are summarized in Figs. 2 and 3. In general, the scatterer system, which is centered on an off-centered particle, should be considered as rotated as well as displaced with respect to the beam system. If the terms defined in the scatterer system are denoted by primed quantities, the transformations between the beam and the scatterer systems will be given as

$$
\begin{aligned}
\mathbf{r}^{\prime} & =[T](\mathbf{r}+\mathbf{d}), \\
\mathbf{r} & =[T]^{-1} \mathbf{r}^{\prime}-\mathbf{d},
\end{aligned}
$$

where $\mathbf{d}$ is the vector defining the displacement of the scatterer from the origin of the beam system, and $[T]$ is the coordinate rotation transformation matrix given as

$$
[T]=\left[\begin{array}{lll}
a_{11} & a_{12} & a_{13} \\
a_{21} & a_{22} & a_{23} \\
a_{31} & a_{32} & a_{33}
\end{array}\right],
$$

If the scattering plane is taken to be the $x z$ plane of the beam system, the elements of $[T]$ are given as

$$
\begin{aligned}
& a_{31}=\sin \theta_{s}, \\
& a_{32}=0, \\
& a_{33}=\cos \theta_{s}, \\
& a_{21}=-q a_{33} / a_{r}, \\
& a_{22}=\left(a_{33} p+s a_{31}\right) / a_{r}, \\
& a_{23}=q a_{31} / a_{r}, a_{r}=\left[\left(q a_{33}\right)^{2}+\left(p a_{33}+s a_{31}\right)^{2}+\left(q a_{31}\right)^{2}\right]^{1 / 2}, \\
& a_{11}=a_{22} a_{33}, \\
& a_{12}=q / a_{r}, \\
& a_{13}=-a_{22} a_{31},
\end{aligned}
$$

and $\theta_{s}$ is the scattering angle which is assumed to be in the $x z$ plane as shown in Fig. 3.

Note that with these coordinate transformations, the plane wave components of the PWS of the laser beam can be transformed to the scatterer system. Applications of such transformations to Eq. (9) give the corresponding plane wave components in the scatterer system,

$$
\mathbf{E}_{i}^{\prime}(p, q)=\mathbf{A}_{i}^{\prime}(p, q) \exp [-i \Delta(p, q)] \exp \left[i k_{i}^{\prime}(p, q) \cdot \mathbf{r}^{\prime}\right],
$$
where

$$
\begin{aligned}
\mathbf{A}_{i}^{\prime}(p, q) & =[T] \mathbf{A}_{i}(p, q), \\
\mathbf{k}_{i}^{\prime}(p, q) & =[T] \mathbf{k}_{i}(p, q), \\
\Delta(p, q) & =\mathbf{k}_{i}(p, q) \cdot \mathbf{d} .
\end{aligned}
$$

So applying the transformation

$$
\left.\begin{array}{rl}
\mathbf{E}_{0} & \rightarrow \mathbf{A}_{i}^{\prime}(p, q) \exp [-i \Delta(p, q)], \\
\mathbf{k} & \rightarrow \mathbf{k}_{i}^{\prime}(p, q), \\
\mathbf{r} & \rightarrow \mathbf{r}^{\prime} \quad \text { and } \quad \mathbf{m} \rightarrow \mathbf{m}^{\prime} .
\end{array}\right\}
$$

In Eq. (10) give the scattered fields due to a single plane wave component of the PWS of the laser beam. These scattered fields are defined in the scatterer system. The corresponding scattered field components in the beam system are then found using the transformations defined in Eq. (14). Finally, the total scattered field due to the incident laser beam is found by superposing these results over the whole $p, q$ plane. The results are ${ }^{9}$ 


$$
\begin{aligned}
\mathbf{E}_{s t}^{(1)}(\mathbf{r})= & \frac{k^{2}}{r} \exp (i k r) \iint_{-\infty}^{+\infty} \mathbf{E}_{i \perp}^{b}(p, q) \exp [-i \Delta(p, q)] \\
& \cdot u\left[k \hat{r}^{\prime}-\mathbf{k}_{i}(p, q)\right] d p d q, \\
\mathbf{E}_{s t}^{(2)}(\mathbf{r})= & \frac{2 k^{2}}{(2 \pi)^{2}} \frac{\exp (i k r)}{r} \iint_{-\infty}^{+\infty} \\
& \cdot \exp [-i \Delta(p, q)] \int_{V_{m}} \frac{u\left(\mathbf{m}^{\prime}+k \hat{r}^{\prime}\right) u\left[\mathbf{m}^{\prime}+\mathbf{k}_{i}^{\prime}(p, q)\right]}{m^{2}-k^{2}} \\
& \cdot\left\{\left(\frac{2}{3} k^{2}+\frac{1}{3} m^{2}\right) \mathbf{E}_{i \perp}^{b}(p, q)\right. \\
& \left.-\left[\mathbf{m}^{\prime} \cdot \mathbf{E}_{i}^{\prime}(p, q)\right] \mathbf{m}_{\perp}^{b}\right\} d^{3} m^{\prime} d p d q,
\end{aligned}
$$

$$
\begin{aligned}
\mathbf{E}_{i}^{b}(p, q) & =[T]^{-1} \mathbf{E}_{i}^{\prime}(p, q), \\
\mathbf{m}^{b} & =[T]^{-1} \mathbf{m}^{\prime}
\end{aligned}
$$

and all primed quantities are defined in the scatterer system. $\mathbf{E}_{s t}^{(1)}(\mathbf{r})$ and $\mathbf{E}_{s t}^{(2)}(\mathbf{r})$ are the complete results of the scattered fields in the first two iterations due to the incident laser beam. The total scattered field due to an incident Gaussian beam is given by

$$
\mathbf{E}_{s t}(\mathbf{r})=\alpha \mathbf{E}_{s t}^{(1)}(\mathbf{r})+\alpha^{2} \mathbf{E}_{s t}^{(2)}(\mathbf{r}) .
$$

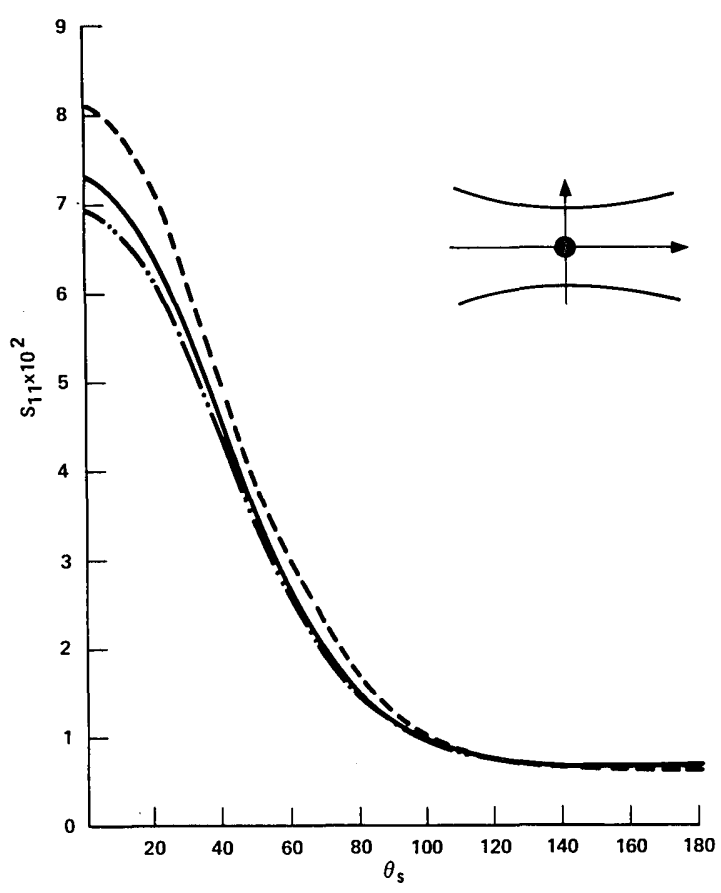

(a)

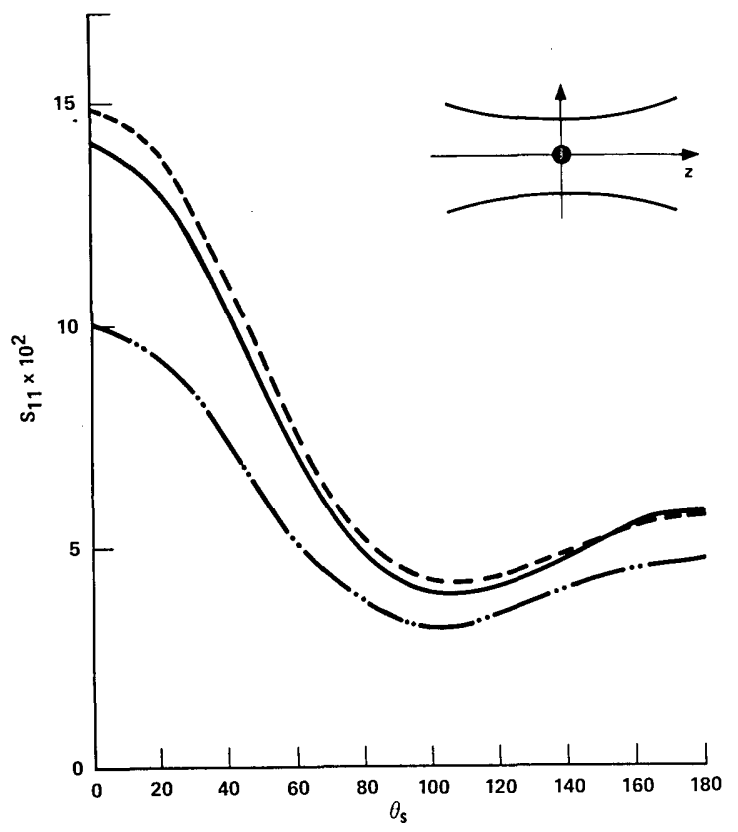

(c)

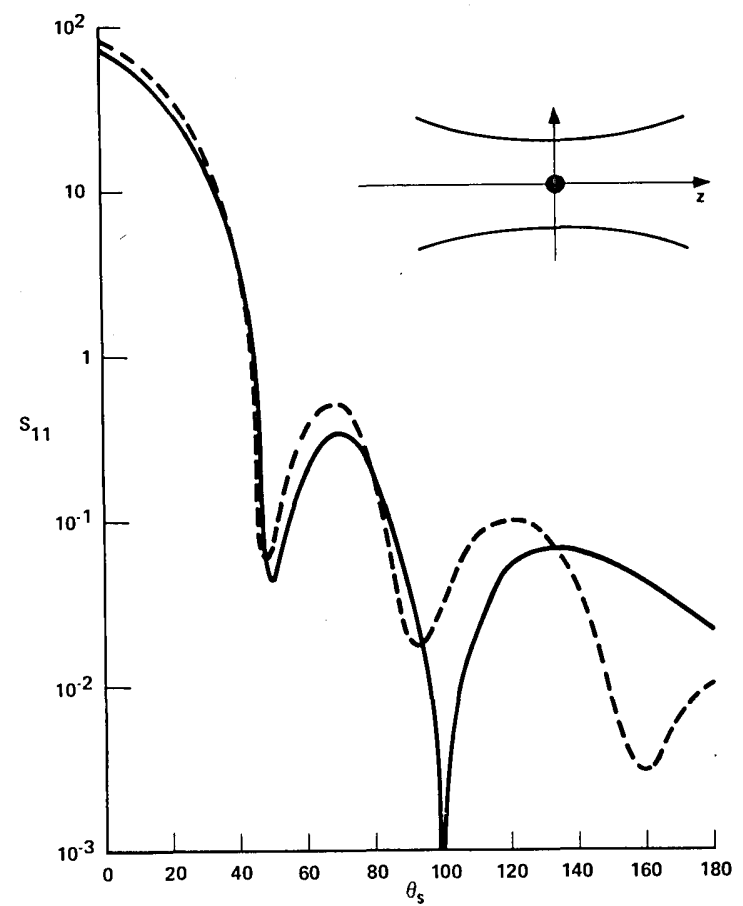

(b)

Fig. 4. Beam scattering patterns for centered spheres (solid lines). In each case, the incident laser beam has a large spot size. Dashed lines give the plane wave results for comparison purposes. The dotted lines represent the first iteration results [if $\mathbf{E}_{s t}^{(2)}(\mathbf{r})$ is taken to be zero]. For all figures $W_{0} / a=5.0$. The scatterer parameters are (a) $k a=$ $1.60, n=1.10$; (b) $k a=5.0, n=1.10$; (c) $k a=1.0, n=1.55$. 
Computations may be carried out in terms of the Stokes parameter $S_{11}$, which is defined as follows:

$$
S_{11}=k^{2} r^{2}\left\{\left[I_{s}(\mathbf{r})\right] /\left[I_{i}(\mathbf{r})\right]\right\},
$$

where $I_{s}(\mathbf{r})$ and $I_{i}(\mathbf{r})$ are, respectively, the scattered and the incident field intensities, i.e.,

$$
\begin{aligned}
& I_{s}(\mathbf{r})=\left|\alpha \mathbf{E}_{s t}^{(1)}(\mathbf{r})+\alpha^{2} \mathbf{E}_{s t}^{(2)}(\mathbf{r})\right|^{2}, \\
& I_{i}(\mathbf{r})=\left(A_{x}^{2}+A_{y}^{2}\right)^{1 / 2},
\end{aligned}
$$

where $\mathbf{E}_{s t}^{(1)}(\mathbf{r})$ and $\mathbf{E}_{s t}^{(2)}(\mathbf{r})$ are given by Eq. (17), and $A_{x}$ and $A_{y}$ have been defined for a Gaussian beam as shown in Eq. (1). Note that in Eq. (20b) maximum intensity of the laser beam (which occurs at origin) is taken to be the incident intensity. By combining Eqs. (17), (19), and (20) and omitting small terms, the following equation is obtained:

$$
\begin{aligned}
S_{11}= & k^{2} \alpha^{2} \iiint \int_{\infty}^{-\infty} \cos \left[\Delta(p, q)-\Delta\left(p^{\prime}, q^{\prime}\right)\right] \\
& \cdot\left\{\hat{\epsilon}_{i \perp}^{b}(p, q) \cdot \hat{\epsilon}_{i \perp}^{b}\left(p^{\prime}, q^{\prime}\right) u\left[k r^{\prime}-\mathbf{k}_{i}^{\prime}(p, q)\right] u\left[k r^{\prime}-\mathbf{k}_{i}^{\prime}\left(p^{\prime}, q^{\prime}\right)\right]\right. \\
& +\frac{\alpha}{\pi^{2}} \int_{V_{m}} u\left[k r^{\prime}-\mathbf{k}_{i}^{\prime}(p, q)\right] \frac{u\left(\mathbf{m}^{\prime}+k r^{\prime}\right) u\left[\mathbf{m}^{\prime}+\mathbf{k}_{i}^{\prime}\left(p^{\prime}, q^{\prime}\right)\right]}{m^{2}-k^{2}} \\
& \cdot\left\{\left(\frac{2}{3} k^{2}+\frac{1}{3} m^{2}\right) \hat{\epsilon}_{i \perp}^{b}(p, q) \cdot \hat{\epsilon}_{i \perp}^{b}\left(p^{\prime}, q^{\prime}\right)-\left[\hat{\epsilon}_{i \perp}^{b}(p, q) \cdot \mathbf{m}_{\perp}^{b}\right]\right. \\
& \left.\cdot\left[\hat{\epsilon}_{i}^{\prime}\left(p^{\prime}, q^{\prime}\right) \cdot \mathbf{m}^{\prime}\right] d^{3} m^{\prime}\right\} d p d q d p^{\prime} d q^{\prime},
\end{aligned}
$$

where $\hat{\epsilon}_{i \perp}^{b}(p, q)$ and $\hat{\epsilon}_{i}(p, q)$ are unit vectors along the electric field vectors $\mathbf{E}_{i \perp}^{b}(p, q)$ and $\mathbf{E}_{i}^{\prime}(p, q)$, respectively.

Numerical examples have been carried out for various particle positions, incident beam spot sizes, and various $k a$ values for spherical scatterers. The applications can easily be extended to other particle shapes using Eq.

(12). For spherical scatterers,

$$
\begin{aligned}
u(\mathbf{v}) & =V_{s} f(a v), \\
V_{s} & =4 / 3 \pi a^{3}, \\
f(a v) & =\frac{3}{(a v)^{3}}[\sin (a v)-(a v) \cos (a v)],
\end{aligned}
$$

where $a$ is the radius of the scatterer.

\section{Numerical Examples}

The first computations were carried out to check if the results obtained in the limit for which the beam waist spot size, $W_{0}$ 's large, were comparable to those for the plane wave scattering case. The comparisons were done for different values of $k a$ and index of refraction $n$. For the case of large $W_{0}$, the scattered field distributions are in close agreement with Acquista's single plane wave scattering results. Figures $4(\mathrm{a}), 4(\mathrm{~b})$, and 4(c) show some examples of these comparisons. In Figs. 4(a) and 4(c), first and second iterations are plotted separately to reveal the advantage of the present approach to the Rayleigh-Gans approximation. The first iteration corresponds to the case in which $E_{s t}^{(2)}(\mathbf{r})$ is taken to be zero. The second iteration is the total result as defined in Eq. (18).

As can be seen from these figures, the case of the scattering by Gaussian beam with large spot size closely resembles that by plane wave case. The major difference is a slight decrease in the scattered amplitude for the beam case. This effect is more observable as the spot size decreases.

The next calculation is carried out to observe the effect of the size of the scatterer and/or the beam spot size on the scattered field of a centered particle. Different ratios of $W_{0} / a$ were used. It is observed that as $W_{0} / a$ is decreased, the scattering pattern shows a shift from $\theta_{s}=0^{\circ}$ to $\theta_{s}= \pm 180^{\circ}$. In other words, the dips and the peaks of the scattering pattern move toward $\theta_{s}= \pm 180^{\circ}$ direction. As the spot decreases, the forward scattered beam is broadened, and its peak is decreased. This effect is more observable as the value of $k a$ increases. As $k a$ increases to a large value, a dip appears in the forward direction. The pattern shift in such cases is as much as 10 degrees for the first dip, and this corresponds to a spot size decrease by a factor of 10 . However, it is seen that the shifts are not linearly dependent on spot size changes. Figures 5(a), 5(b), 5(c), and 5(d) summarize the effects of changing $W_{0} / a$ ratios on the scattered field. Figures 5(c) and 5(d) are included to show the effects of a beam waist spot size which is less than the scatterer radius, that is, the $\left(W_{0} / a\right)<1$ case. As seen in Fig. 5(c) the forward peak is relatively flat for $\left(W_{0} / a\right)=0.65$, and in Fig. 5(d) the forward scattered field begins to show a dip at $\theta_{s}=0^{\circ}$ for $\left(W_{0} / a\right)=0.50$. In earlier figures these dips did not appear. This is due to the fact that for the parameters chosen the diffraction limit of the beam was exceeded in these cases. The flattening and the dipping of the pattern in the forward scattering direction and the shifting of the scattered energy toward $\theta_{s}= \pm 180^{\circ}$ are caused by the interference effects of fields inside the particle. These results show strong resemblance to the fringe pattern changes and shifts obtained from a Fabry-Perot etalon. ${ }^{10}$ Using the geometrical optics principles, the pattern shifts or the pattern expansions may be explained by the changes of the incident beam phase front curvature. According to the beam expression given by Eq. (1), one notes that decreasing the spot size $W_{0}$ increases the beam phase front curvature, $1 / R(a)$, on the particle surface. As this phase curvature increases, it becomes better matched to the particle surface. Consequently fewer fringes are expected. This also causes the scattered beam pattern to expand. Similar results for centered spheres were also found by Tsai and Pogorzelski. ${ }^{11}$

However, an obvious question arises with the appearance of a dip in the forward peak. The above etalon example could also explain this, but only if a change in the round-trip phase delay along the particle axis due to spot size changes is assumed. If the beam expression of Eq. (1) is considered, it is seen that, in fact, the phase of the beam does depend on the waist spot size through the last exponential term in Eq. (1b). A simple derivation shows that for a complete destructive interference along the $z$ axis of the sphere, one should have

$$
4 k_{0} n a+4 \tan ^{-1}\left(a / z_{0}\right)=(2 h+1) \pi,
$$

where $h$ is any integer. Using Eq. (1c) for $z_{0}$ and rearranging gives the relation, 


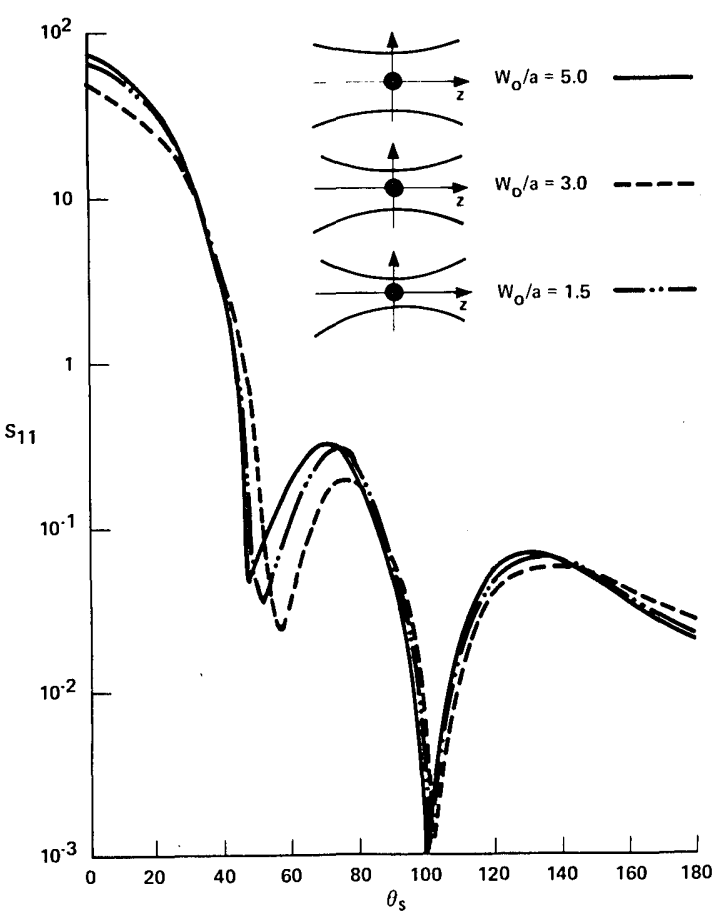

(a)

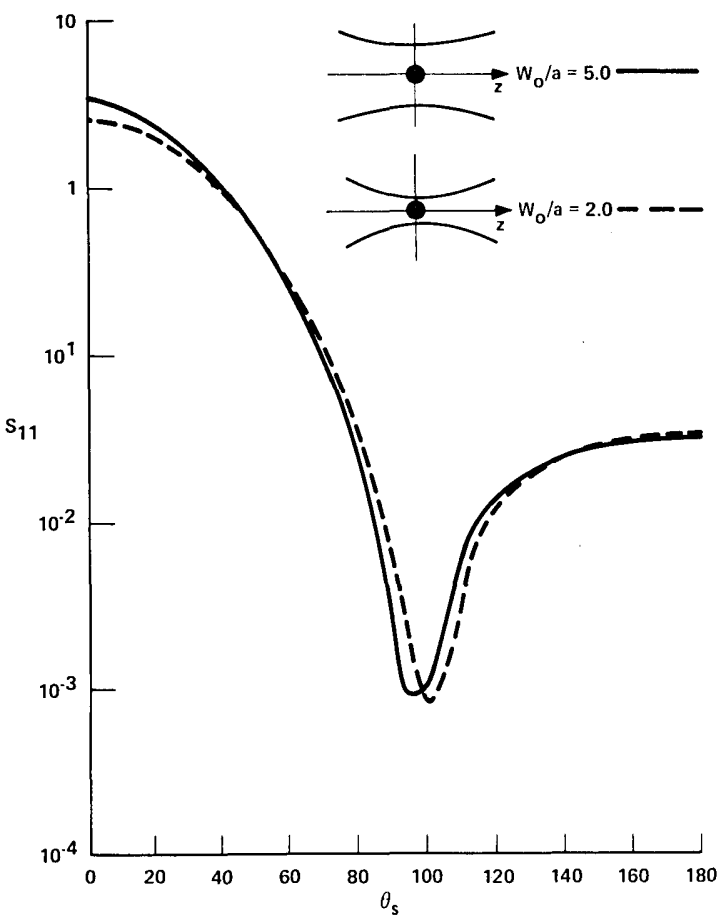

(b)

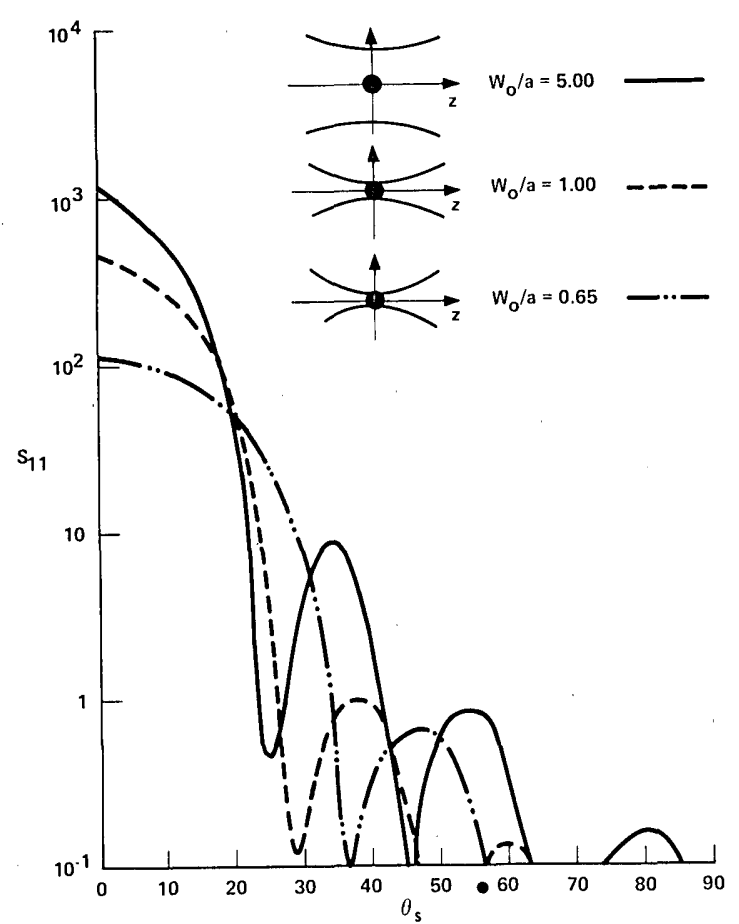

(c)

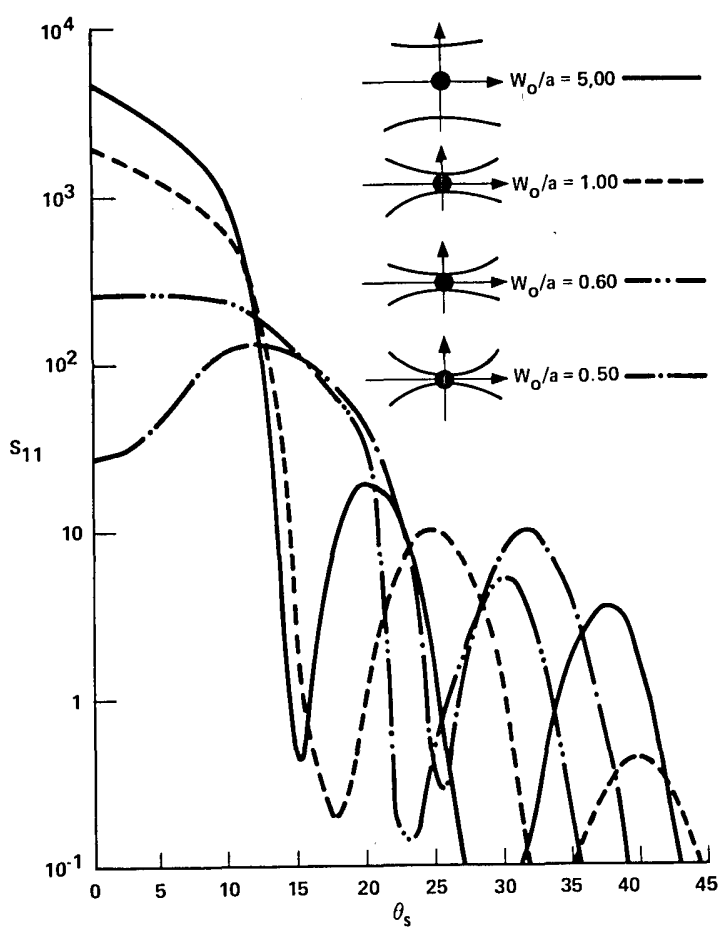

(d)

Fig. 5. Beam scattering patterns showing the effect of decreasing beam waist spot size $W_{0}$ for centered spherical scatterers. The expansion of the pattern for smaller spot sizes is easily observable. The scatterer parameters are: (a) $k a=5.0, n=1.10$; (b) $k a=3.0, n,=1.10$; (c) $k a$ $=10.0, n=1.05$; (d) $k a=15.0, n=1.03$. The spot sizes are indicated in figures in $W_{0} / a$ ratios. 


$$
\frac{W_{0}}{a}=\left[\frac{2}{n k_{0} a \tan \left(\frac{2 h+1}{4} \pi-n k_{0} a\right)}\right]^{1 / 2},
$$

using this equation for the parameters of Fig. 5(d) shows that the only value of the $\left(W_{0} / a\right)$ ratio which may cause a complete destructive interference along the $z$ axis within the particle is 0.28 . This corresponds to a beam phase front curvature radius at the scatterer surface of about $1.60 a$.

The above reasons explain the pattern shift, forward peak flattening, and dipping. The decrease of the over-all magnitude of the pattern is due to the fact that the more the beam is focused, the smaller is the amplitude of the incident field. This is simply caused by taking the peak amplitude of the fields being constant, i.e., unity. In practical experiments, this will not be true. In other words, focusing the laser beam will increase the field magnitudes near the origin, and thus increase the magnitude of the scattering pattern. These effects can be taken into account by a simple proportional increase of the patterns given in this section.

Another set of calculations was done to find the effects of off-centered particles. Some results of this set are shown in Figs. 6. Figures 6(a) and 6(b) show the effect of changing the position of the particle in the $z=$ 0 plane. Changing the particle position in this plane reduces the intensity of the scattered fields, but does not change the scattering pattern. The latter effect is due to the Gaussian amplitude distribution of the incident beam. Figure 6(a) shows that as the particle is moved from the origin $(0,0,0)$ to the point $(2,1 / 2,0)$ or to $(4,3,0)$, the parameter $S_{11}$, at the forward peak, is reduced by

$$
\frac{S_{11,(0,0,0)}}{S_{11,(2,1 / 2,0)}}=1.71, \quad \frac{S_{11,(0,0,0)}}{S_{11,(4,3,0)}}=8.3
$$

for each case. These values support the reasoning given above, because the intensity of the beam at the origin $(0,0,0)$, is greater than its value at the points $(2,1 / 2,0)$ and $(4,3,0)$ by

$\frac{I_{\text {inc },(0,0,0)}}{I_{\text {inc, }(2,1 / 2,0)}}=\exp \left[2\left(d_{x}^{2}+d_{y}^{2}\right) / W_{0}^{2}\right]=1.65, \quad \frac{I_{\text {inc },(0,0,0)}}{I_{\text {inc, },(4,3,0)}}=7.38$, which are close to the respective values obtained for $S_{11}$. Similar calculations for the parameters of Fig. 6(b) show that

$$
\begin{array}{ll}
\frac{S_{11,(0,0,0)}}{S_{11,(2,2,0)}}=1.88, & \frac{I_{\text {inc, }(0,0,0)}}{I_{\text {inc, }(2,2,0)}}=1.90, \\
\frac{S_{11,(0,0,0)}}{S_{11,(4,4,0)}}=12.48, & \frac{I_{\text {inc, }(0,0,0)}}{I_{\text {inc, }(4,4,0)}}=12.94 .
\end{array}
$$

The decrease in the magnitude of $S_{11}$ is nearly equal to the decrease of the incident intensity caused by offcentering the particle.

The scattering pattern caused by an off-centered particle in $z=0$ plane is found to be exactly symmetrical. Although this is unrealistic because of propagation characteristics of a Gaussian beam, it is somewhat predictable because the present approach of this study is just an iterative approximation for the scattering problem. The asymmetry for $z \neq 0$ case as seen from
Fig. 6(c) is relatively small. However, one expects increasing asymmetry as the particle moves parallel to the $z$ axis toward the $z=0$ plane. This last point, again, can be reasoned from the beam propagation characteristics.

Another result is the change of the scattering pattern caused by a change of position of an off-centered particle on a circular path, which is centered on the origin and lies in the $z=0$ plane. That is, a particle located at $(1,0,0)$ gives different scattering pattern than a particle at $(\sqrt{ } 2, \sqrt{ } 2,0)$, although they are at equal distances from the origin. This assumes that the other parameters of the scattering are fixed. The above effect is a beam scattering characteristic and cannot be observed in the far-field behavior of the single plane wave scattering.

If the scatterer is off-centered at a value $z \neq 0$, and for nonzero $d_{x}$, and $d_{y}$ displacements, the scattering pattern is asymmetric. This is because the incident field evaluated at the center of the scatterer has a phase front which is directed in a different way than the $z$ axis. This seems to be the cause of the asymmetry of the pattern for displacements away from the $z=0$ plane. Figure 6(c) gives an example of this. The difference between the $S_{11}$ values for both signs of the scattering angle $\theta_{s}$ from $0^{\circ}$ to $180^{\circ}$ is plotted on a different scale to show the asymmetric distribution.

The results of our earlier study ${ }^{1}$ show that if the displacement $\left(d_{x}, d_{y}, d_{z}\right)$ is changed to $\left(-d_{x},-d_{y},-d_{z}\right)$, the scattering pattern completely repeats itself. However, if $\left(d_{x}, d_{y}, d_{z}\right)$ is changed to $\left(-d_{x},-d_{y}, d_{z}\right)$, one gets inverted asymmetrical distribution compared with the original case. If $d_{z}=0$, the pattern is the same and symmetrical for all the cases given above. The computations of the present work agreed with these results. This behavior can be predicted by considering the correlation term $\cos \left[\Delta(p, q)-\Delta\left(p^{\prime}, q^{\prime}\right)\right]$ of Eq. (29). As stated there, $\Delta(p, q)$ is a linear function of displacement given by $\Delta(p, q)=k\left(p d_{x}+q d_{y}-s d_{z}\right)$. So a change of $\left(d_{x}, d_{y}, d_{z}\right)$ to $\left(-d_{x},-d_{y},-d_{z}\right)$ does not change the scattering pattern because the cosine function is even. However, as explained before, this behavior is not realistic, and the error comes from the inherent assumptions of Rayleigh type scattering solutions about the behavior of fields inside the scatterer.

The computation of Eq. (21) to obtain scattered field patterns was carried out on an IBM 360 using a Gaussian quadrature scheme. Since the integrand contains a relatively localized Gaussian envelope function, very little computer time was needed. For centered particles the CPU time was about $10 \mathrm{sec}$, and for off-centered particles the time increased to a maximum of $40 \mathrm{sec} /$ plot.

\section{Conclusions}

Results of the previous section showed that if the maximum dimension of the scatterer is $a$, and the waist spot size of the beam is $W_{0}$, under the conditions that the scatterer is centered on the beam waist, and $a \lesssim$ $\left(W_{0} / 5\right)$, the scattering patterns obtained have slight differences (less than $5 \%$ ) from the single plane wave scattering patterns. However, if either of the above 


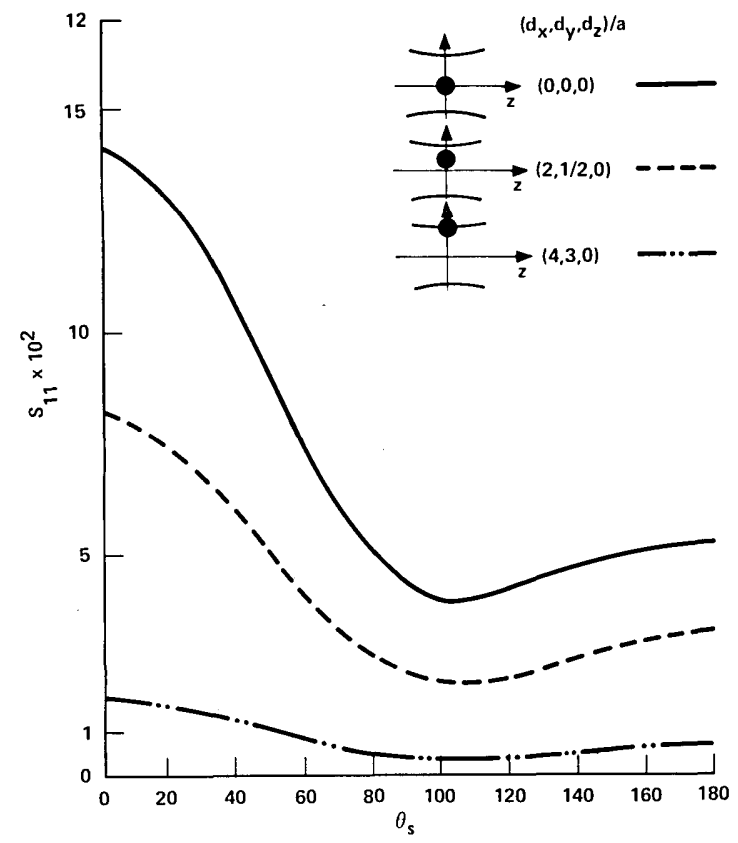

(a)

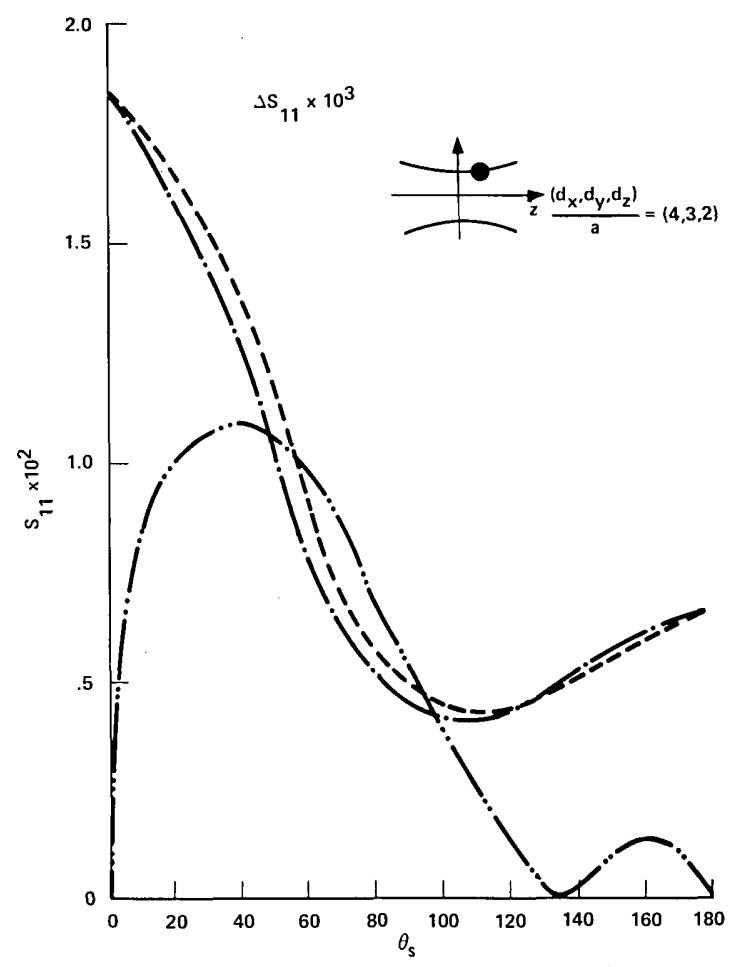

(c)

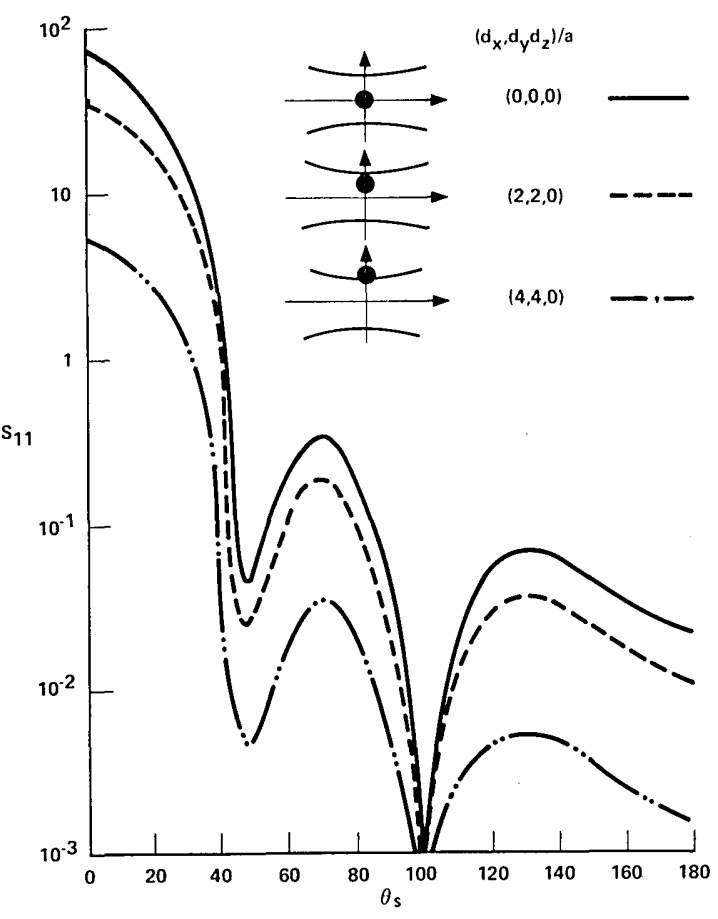

(b)

Fig. 6. Beam scattering patterns for off-centered spherical scatterers; (a) and (b) are for scatterers off-centered in the $z=0$ plane. The magnitude reduction in the pattern is proportional to the incident beam intensity decreases due to off-centering. (c) is for scatterers off-centered out of the $z=0$ plane. For this case, the difference between the $S_{11}$ values for different directions of the scattering plane is shown by the dotted curve to reveal the asymmetry of the pattern. The beam and the scatterer parameters for the figures are as follows: (a) and (c) $W_{0} / a=5.0, k a=1.0, n=1.55$; (b) $W_{0} / a=5.0, k a=5.0$, $n=1.10$, and the displacements of scatterers from the beam system origin are given in the figures in terms of $\left(d_{x}, d_{y}, d_{z}\right) / a$ ratios. For (c), $S_{11}$ values for positive and negative scattering angles are given by dashed curves without and with dots, respectively. The difference between these two cases is plotted by the dotted curve.

conditions is not satisfied, the patterns of the beam scattering showed great differences compared with the single plane wave results. These differences are summarized below.

Decreasing the spot size of the beam relative to the dimensions of the scatterer which is centered on the beam waist caused three common changes in the scat- tering pattern in most cases. First there was the decrease in the over-all magnitude of the scattered field. This is simply because in each case the maximum amplitude of the beam is taken to be unity. So, the sharper the beam is focused, the less is the total energy contained in the beam. Second was the flattening of the forward peak and even an appearance of a dip in the 
center of the peak for very small spot sizes. This effect was due to the fact that the curvature of the beam becomes a better fit for the scatterer surfaces when the spot size gets smaller. When the curvature becomes a closer fit to the surface of the particle, the fields start interfering within the particle. The calculations done in previous sections showed that the dips in the forward peak occurred close to some specific beam parameters, which satisfied the conditions of a destructive interference on the $z$ axis of the scatterer. The other change in the pattern which is caused by a decrease in the spot size is the expansion of the pattern, i.e., the shift of the dips and the peaks from forward to the backward scattering directions. The explanation of this effect is also found to be the better fit of the phase front curvature of the beam to the scatterer surface. These points are explained in a more detailed way in the previous section.

Off-centered particles created two different situations. First, changing the particle position in the $z=$ 0 plane away from the origin caused a smooth decrease in the scattering pattern. The reason for this is the Gaussian amplitude distribution of the incident laser beam (see the previous section for numerical examples). Second, moving an off-centered scatterer out of the $z$ $=0$ plane parallel to the $z$ axis caused an asymmetry in the scattering pattern.
This work was supported in part by the National Science Foundation.

\section{References}

1. L. W. Casperson, C. Yeh, and W. F. Yeung, Appl. Opt. 16, 1104 (1977).

2. L. W. Casperson and C. Yeh, Appl. Opt. 17, 1637 (1978).

3. K. S. Shifrin, Scattering of Light in a Turbid Medium (Nauka, Moscow, 1951; NASA, Washington, D.C., 1968), Technical Translation TTF-477.

4. C. Acquista, Appl. Opt. 15, 2932 (1976).

5. A. Yariv, Quantum Electronics (Wiley, New York, 1975).

6. J. W. Goodman, Introduction to Fourier Optics (McGraw-Hill, New York, 1968).

7. P. C. Clemmow, Plane Wave Spectrum Representation of Electromagnetic Fields (Pergamon, London, 1966).

8. W. H. Carter, J. Opt. Soc. Am. 62, 1195 (1972).

9. S. Colak, "Focused Laser Beam Scattering by Stationary and Moving Particles," Ph.D. Thesis, U. California, Los Angeles (1978).

10. A. Yariv, Introduction to Optical Electronics (1971).

11. W. C. Tsai and R. J. Pogorzelski, J. Opt. Soc. Am. 65, 1457 (1975).
June

? $\quad$ AAS Meeting, Wellesley, Mass. L. W. Frederick, P.O. Box 3818, Univ. Station, Charlottesville, Va. 22903

17-19 Trends and Applications Symp., NBS, Gaithersburg $S$. Watkins, B212 Technol. Bldg., NBS, Washington, D.C. 20234

21-25 1st Australasian Landsat Conf., Sydney J. Davies, P.O. Box 136, N. Ryde, NSW, 2113, Australia

21-25 Laser Safety course, Washington, D.C. Laser Inst. of Am., P.O. Box 9000, Waco, Tex. 76710

21-25 Fast Ion Transport in Solids-Electrolytes and Electrodes, internat. conf., Lake Geneva, Wisc. P. Vashishta, Solid State Sci. Div., ANL, Argonne, Ill. 60439

22-25 SPIE Huntsville Electro-Optical Tech. Symp. and Workshop, Von Braun Civic Ctr., Huntsville, Ala. SPIE, P.O. Box 10, Bellingham, Wash. 98225

Laser Optics course, Dallas Laser Inst. of Am., P.O. Box 9000, Waco, Tex. 76710

European Optical Comm., 1st Conf., Bad Harzburg K.-J. Rosenbruch, PTB, Bundesallee 100, D-3300 Braunschweig, Germany

Shock Waves in Condensed Matter, APS topical conf., Washington State U. G. E. Duvall, Phys. Dept., WSU, Pullman, Wash.

Applications of Ferroelectrics, IEEE internat. symp. Sheraton Ritz Hotel, Minneapolis S. T. Liu, Honeywell Corp. Res. Ctr., 10701 Lindale Ave., S., Bloomington, Minn. 55420

2nd Joint MMM-Intermag Conference, New York $R . M$. Josephs, Sperry Univac Computer Systems, P.O. Box 500, Blue Bell, Pa. 19422

18-21 USNC/URSI/IEEE, mtg., Seattle A. Ishimaru, Dept of Electrical Eng., FT-10, U. of Washington, Seattle, Wash. 98195

18-22 Laser '79 Exhibition and Congress, West Germany $C$ Werner, DFVLR, Inst. für Physik der Atmosphare, 801 Oberpfaffenhofen, Post Wessling/OBB, German Federal Republic

18-22 Carbon Dioxide Lasers course, Los Alamos Laser Inst. of Am., P.O. Box 9000, Waco, Tex. 76710

30-1 June IEEE/OSA Conference on Laser Engineering and Applications, Washington Hilton Hotel J.W. Quinn, OSA, 2000 L St. N.W., Washington, D.C. 20036 